\title{
Criminologie
}

\section{Les origines de la prison de Bordeaux}

\section{Pierre Landreville et Ghislaine Julien}

Volume 9, numéro 1-2, 1976

L’emprisonnement au Québec

URI : https://id.erudit.org/iderudit/017049ar

DOI : https://doi.org/10.7202/017049ar

Aller au sommaire du numéro

Éditeur(s)

Les Presses de l'Université de Montréal

ISSN

0316-0041 (imprimé)

1492-1367 (numérique)

Découvrir la revue

Citer cet article

Landreville, P. \& Julien, G. (1976). Les origines de la prison de Bordeaux.

Criminologie, 9(1-2), 5-22. https://doi.org/10.7202/017049ar d'utilisation que vous pouvez consulter en ligne.

https://apropos.erudit.org/fr/usagers/politique-dutilisation/ 


\section{LES ORIGINES \\ DE LA PRISON DE BORDEAUX}

Pierre Landreville Ghislaine Julien

«Aujourd'hui comme hier, le manque de souplesse est évident dans l'architecture d'un grand nombre de prisons au Canada. En conséquence, nous sommes encombrés de grands édifices de pierre et d'acier, certains érigés au siècle dernier et fort peu faciles à adapter à un programme moderne de traitement."

Rapport du Comité canadien de la réforme pénale et correctionnelle (1969 p. 349).

Tous ceux qui ont jeté un coup d'œil sur les prisons canadiennes et québécoises sont frappés, comme l'ont été les membres du Comité Ouimet, par le lourd héritage que nous a laissé le $19^{\mathrm{e}}$ siècle. Nous sommes en effet «encombrés de grands édifices de pierre et d'acier, certains érigés au siècle dernier..." Cette constatation est d'autant plus déconcertante lorsqu'on s'aperçoit que la majorité de ces prisons du $19^{\mathrm{e}}$ siècle et du début du vingtième ne sont en réalité que des répliques d'édifices conçus au début du $19^{\mathrm{e}}$ siècle.

La prison de Montréal, communément appelée prison de Bordeaux, a été construite de 1908 à 1912 et est une des seules prisons au Canada de type Pennsylvanien'.

En plus de s'inspirer d'une philosophie et d'une architecture presque centenaire, la prison de Bordeaux est assez unique en son

1. Du nom du premier pénitencier, érigé à Philadelphie en 182l, où l'on favorisait l'isolement cellulaire. 
genre en Amérique du Nord puisque le système Pennsylvanien a été surtout en vogue en Europe au $19^{\text {e }}$ siècle et n'a presque pas influencé la construction de prisons en Amérique du Nord.

Aussi, il nous semble important de découvrir dans quel contexte fut érigé cet établissement qui, depuis 1912, a été la principale prison " du district judiciaire de Montréal. Nous tenterons donc de voir, dans ces premières notes de recherche, quel était le contexte pénologique au Québec au siècle dernier, les circonstances immédiates qui ont favorisé l'érection d'une nouvelle prison, les principaux acteurs de cette réalisation ainsi que quelques événements associés à la construction de la prison.

\section{A. CONTEXTE PÉNOLOGIQUE DU $19^{\circ}$ SIÈCLE}

L'on sait que le système pénitentiaire, tel que nous le connaissons aujourd'hui, a pris naissance aux États-Unis au début du siècle dernier. Auparavant, l'emprisonnement existait surtout pour les prévenus, les condamnés qui attendaient leur châtiment (peine de mort, mutilation, déportation) les vagabonds et les débiteurs insolvables. D'une part en effet, inspiré par l'ouvre de John Howard", les quakers Pennsylvaniens élaborèrent une nouvelle philosophie pénale qui se concrétisa en 1821 par la construction du Eastern Penitentiary à Philadelphie. Le principe de base de ce système "Pennsylvanien" consiste dans l'isolement individuel du détenu de jour et de nuit. On croyait que la méditation conduirait au repentir et par lui à l'amendement du coupable. De plus, le régime cellulaire réalise la séparation totale et complète des détenus et, de ce fait, évite le danger de la promiscuité et préserve de la contamination. Du point de vue architectural, le système Pennsylvanien est caractérisé par des grandes cellules, à portes pleines, dont les fenêtres donnent sur l'extérieur.

D'autre part, à la même époque (1825) l'on construisait à Auburn dans l'État de New-York, un pénitencier dont le principe de base, à la différence du système Pennsylvanien, repose sur l'emprisonnement en commun pendant le jour avec isolement pendant la nuit. Dans ce régime mixte, les détenus sont soumis, pendant les moments où ils vivent et travaillent en commun, à

2. Bordeaux a été le principal établissement provincial pour les prévenus et les détenus condamnés à des peines de moins de deux ans.

3. En 1777, John Howard écrivit un livre très célèbre : State of Prisons in England and Wales. 
la règle du silence. Ce système est caractérisé par de petites cellules, dos à dos, sans fenêtres mais dont les portes sont ajourées pour laisser passer l'air et la lumière du jour.

Après 1830, les débats entre les tenants de l'un et l'autre de ces systèmes furent très nombreux et très importants au point de vue pénologique. Ces innovations et ces échanges d'idée attirèrent en Amérique de nombreux visiteurs, observateurs et enquêteurs étrangers. Entre autres, en 1831, deux français, Alexis de Tocqueville et Gustave de Beaumont furent envoyés pour faire une étude comparative de ces deux systèmes. Leur rapport officiel publié à Paris en 1833 sous le titre "du système pénitentiaire aux États-Unis et de son application en France» est un des principaux documents sur la comparaison de ces deux systèmes. Les auteurs signalèrent leurs préférences théoriques pour le système Pennsylvanien. À la même époque, les anglais envoyèrent William Crawford visiter les États-Unis pour y voir de près ces nouvelles expériences pénologiques. Dans son rapport qu'il a remis en 1834 et qui fut imprimé en 1835, William Crawford préconisa lui aussi la mise sur pied d'un système Pennsylvanien en Angleterre. Ses recommandations aboutirent à la construction de la prison Pentonville à Londres, prison qui fut l'une des plus copiées en Europe. De son côté, la France adopta elle aussi, après de longs débats et malgré l'opposition de Charles Lucas ${ }^{4}$, le système Pennsylvanien.

Au Bas-Canada, on est très au fait de ces nouveaux courants de pensée pénologique américains et, dès 1834 , la Chambre du Bas-Canada dépêcha deux commissaires, $M$. Dominique Mondelet, et $\mathbf{M}$. John Neilson pour visiter les pénitentiers et s'enquérir des systèmes disciplinaires qui y sont en vigueur.

... Deux commissaires... dont le devoir sera de se transporter dans les États-Unis d'Amérique, pour y visiter les principaux centres pénitentiaires, constater les différents systèmes de discipline que l'on a adoptés dans de telles prisons et les règlements faits sur le gouvernement et la régie intérieure d'icelles, et de se procurer tels plans, estimations, publications, documents qu'ils croiront les mieux adaptés afin de promouvoir les objets de leur mission, et de mettre la législature en état ci-après de pourvoir efficacement à l'établissement d'un système pénitentiaire pour la discipline des prisons dans cette province, de la manière qui pourra le mieux promouvoir le bien public..."

4. Voir à ce sujet André Normandeau (1970).

5. Acte pour autoriser la nomination de commissaires à l'effet d'obtenir les renseignements nécessaires, préparatoirement à l'introduction du système pénitentiaire 
A leur retour, les commissaires recommandent chaudement l'adoption du système d'emprisonnement tel que pratiqué à Philadelphie. Ce faisant, ils rejettent sans beaucoup de discussions le régime "Auburnien». Selon eux, seul l'isolement total assure la protection de la société et permet d'espérer la réforme du délinquant. Le système de Philadelphie «... à l'effet de le rendre (le criminel) plus soumis, et d'effectuer une plus grande réforme dans ses mœurs et ses habitudes..." Ils ajoutent que le seul avantage du système Auburn, s'il y en avait un, est la possibilité de retirer un plus grand profit du travail des détenus, mais que cette possibilité peut attirer de nombreuses critiques.

... Le système Auburn a occasionné des plaintes très générales parmi les fabricants et les artisans, par suite de la concurrence qu'ils ont à soutenir contre les prisons d'État, dans les branches de commerce et d'industrie qu'ils exercent; que ces plaintes soient fondées ou non, il importe que l'on ne donne aucune prise à la malveillance contre des établissements de bienveillance qui ne font que naître... (Mondelet, D. Nelson J. 1835).

Immédiatement, de nombreuses protestations se sont élevées contre les conclusions des commissaires. Un Comité spécial présidé par M. E. Rodier est formé en 1835, pour étudier la question. Les membres du comité ont tenu à entendre des spécialistes de la question. Amury Girod, philosophe et aventurier qui prit part subséquemment à la bataille de St-Eustache en 1837, au côté du $\mathrm{D}^{r}$ Chenier, fut l'un d'eux. Il dû impressionner fortement les membres du Comité puisque sa conception de la peine orienta tous les propos du Comité.

... La société... n'administre la justice que pour maintenir les droits naturels de chaque individu, qui ne saurait les conserver sans l'assistance de ses semblables. Le corps social décide en effet si un de ses membres est devenu l'ennemi de tous par ses crimes; le corps social le bannit de son sein ou le punit autrement; mais il n'est juge et arbitre que de ses actions extérieures, avec son intérieur, sa croyance, sa conscience, elle n'a ni ne saurait rien avoir à faire. Je ne veux pas conclure de là que la société ne doive pas s'intéresser à la réforme morale du criminel, mais seulement établir le droit de tout homme libre ou non pour n'avoir pour juge de ses pensées, de sa conscience, de ses opinions que Díeu seul. Sans doute, il est à désirer qu'on puisse réussir à réformer le cœur d'hommes pervers, sans doute il est nécessaire de prendre

pour la discipline des prisons en cette province «18 mars 1834», 4 Guillaume IV, ch. 10 , art. ?. 
tous les moyens légitimes de le faire, mais quant à la chance de succès dans cette entreprise, elle semble moins grande qu'on est disposé à le croire..."

Par son argumentation, il tendait à saper celle des commissaires Mondelet et Neilson qui avaient fait de la réforme de l'âme du détenu leur critère principal. M. Girod, un ardent défenseur du système Auburnien, alla loin dans ses propos. Non seulement il affirma que les prétentions des partisans du système Pennsylvanien étaient de «douces illusions" mais que seules celles du système d'Auburn étaient sensées :

le système d'Auburn ne réussit pas non plus à changer le cœur des criminels; mais moins maltraités par la société, plus protégés par elle dans leur affection et leur espérance, ils se soumettent à ses lois, parce que sans la soumission à ces lois, ils savent très bien, qu'ils ne sauraient rester dans cette société qu'ils n'abordent pas, mais qu'ils regrettent, mais qu'ils rappellent de tout leur voux...

Le Comité opta pour le système «qui offrait le plus de chances d'opérer la réforme du criminel». L'un des arguments principaux en faveur du système Auburnien fut le suivant :

... Le prisonier de Cherry-Hill (Pennsylvanie) n'ayant point les moyens de faire le mal, sa conduite paisible et ínoffensive ne peut être considérée comme une vertu acquise par l'application du système de travail solidaire. Le prisonnier d'Auburn, au contraire, travaillant en commun peut enfreindre l'obligation du silence à laquelle il est assujetti. Cependant, cela ne lui arrive presque jamais et il contracte ainsi des habitudes d'obéissance et de soumission qui le suivront à son retour dans la société (Rodier 1836).

Malheureusement, ce projet ne fut jamais adopté. La révolte des patriotes vint, entre autres, y mettre un terme. Cependant, ces extraits nous démontrent clairement que les débats pénologiques de l'époque avaient lieu au Québec dans les mêmes termes et aux mêmes moments qu'ils avaient lieu en Europe et aux États-Unis.

Quelques années plus tard, en 1859, les nouveaux inspecteurs des prisons et des asiles revinrent à la tâche pour convaincre une fois de plus la Législature de la nécessité d'opérer la réforme des

6. Témoignage de M. Amury Girod devant M. Edward Rodier. président du comité spécial de la Chambre (17 février 1836, in : Continuation de l'appendice au ISe volume des journaux de la Chambre d'assemblée du Bas-Canada, $(1835,1836)$ vol. 3, appendice F.F.F. section B.

7. Témoignage de M. Amury Girod, op. cit. 
prisons. Eux aussi, montrèrent une préférence marquée pour le système Auburnien.

Dans les prisons centrales, on pourrait adopter, suivant le cas, le système pénitentiaire du travail en commun, mais en silence, et le système agrégé sans le silence mais avec classification des moralités; Pencellulement solitaire de nuit serait maintenant partout, sauf le cas de maladies graves et autres cas spéciaux et la réclusion solitaire de jour et de nuit pourrait servir de moyens disciplinaires pour les périodes de peu de durée... (Nelson W.D. et al., 1859).

Le Québec continue d'être perméable aux nouveaux courants pénologiques. Ainsi, en 1868 , les membres du nouveau bureau des inspecteurs de prisons d'asiles d'aliénés de la province de Québec, créé en 1867, propose un autre système d'emprisonnement. Selon eux, le système le plus parfait est celui de la «communication au rachat d'une partie de la peine et celui des récompenses" tel que pratiqué en Irlande.

Ce système... est appuyé sur ce mot magique "qui fait vivre» comme le dit le proverbe l'espérance.

Ceci s'opère en mettant autant que possible, le sort du prisonnier entre ses propres mains, et en le rendant capable, par son industrie et sa bonne conduite, de se faire petit à petit une meilleure position...

L'espérance de voir sa peine abrégée et celle de se créer un petit capital qu'on lui remettra à sa sortie de prison, est donc le mobile et l'agent qui portent le détenu dans les prisons d'Irlande à se rêformer et à faire un bon citoyen...

Encore une fois, les Québécois étaient très au fait des nouvelles réformes pénologiques. En effet, le système progressif irlandais qui est à l'origine de la libération conditionnelle telle que nous la connaissons aujourd'hui, avait été mis sur pied en Irlande en 1854 par Sir Walter Crofton. Il avait appliqué de façon originale le mark system de Maconochie en insistant sur la période de transition et le ticket of leave qui avait été introduit en Angleterre et en Irlande par le Penal Servitude Act de 1853.

\section{B. LA SITUATION AU PIED-DU-COURANT}

En 1836, l'on avait inauguré à Montréal, une nouvelle prison, la prison du Pied-du-courant. L'établissement qui recevait préve-

8. Premier rapport annuel du bureau des inspecteurs des prisons, asiles, pour les années 1867 et 1868, in : Documents de la session 1869, vol. 1-2, doc. 23. 
nus, détenus, débiteurs, vagabonds et mêmes malades mentaux des deux sexes, avait été, semble-t-il fort mal conçu.

On peut, en effet, lire dans un rapport de 1835-1836 :

Nous n'avons vu aucun appareil de cuisine, et rien non plus pour laver et fumiger les hardes et lits des prisonniers; point d'infirmerie pour les malades, enfin rien qui puisse nous engager à recommander ce bâtiment comme prison publique...."

Pour sa part, M. C.A. Vallée, dernier gouverneur de la prison du Pied-du-courant de 1891 à 1912 faisait ses commentaires au sujet de l'édifice.

Ces plans et l'édifice lui-même, étaient surtout remarquables par l'absence de toutes techniques pénitentiaires et le dédain absolu, (ou l'ignorance) des principes les plus élémentaires de l'hygiène, de la surveillance et de la discipline... ${ }^{10}$

De fait, durant toute l'existence de cet établissement, l'on s'est plaint de la surpopulation et des conditions de détention. Au début du $20^{*}$ siècle, l'on constatait aussi que l'édifice était très détérioré physiquement.

Ces facteurs en ont incité plusieurs à demander l'agrandissement ou la construction d'une nouvelle prison et cela dès les premières années de l'établissement.

Comme le signale Boyer (1966), dès l'ouverture de la prison en 1837 et en 1838, les arrestations furent si nombreuses, lors du soulèvement des patriotes, qu'on a dû réoccuper la vieille prison et aménager en geôle le magasin Fry à la pointe à Callières. Le gouverneur de la prison a dû faire face aux problèmes épineux d'entasser plus de cinq cents détenus à la fois dans une prison qui, une fois terminée, ne pouvait en contenir que 225 (p. 448).

Le problème du surpeuplement se présente avec beaucoup d'acuité en 1869, car selon les inspecteurs des prisons et asiles de la province de Québec :

Ả Montréal, outre la mauvaise division, à la prison elle-même est près de la moitié trop petite. Le mal y est si grand que

9. Rapport de MM. Clarke et Appleton, au sujet de la nouvelle prison à Montréal, in : Journaux de la Chambre d'assemblée du Bas-Canada (1835-36), p. 218-219.

10. C.A. Vallée, "1913" Note historique, in : Rapport de C.A. Vallée sur la nouvelle prison de Montréal à Bordeaux, document dactylographié 26 juillet 1913. 
nous croyons que c'est un devoir impérieux pour nous de presser l'autorité d'y apporter remède le plus tôt possible. Il faut de toute nécessité agrandir la prison actuelle de près de la moitié, et pourvoir à la construction d'un mur de clôture autour des 14 arpents de terre attachés à l'établissement ou bien adopter le plan que nous proposons en parlant des prisons centrales..."

Le même problème est soulevé de nouveau en 1898 par le gouverneur M. C.A. Vallée dans une lettre à l'inspecteur des prisons M. A. de Martigny. M. Vallée affirme qu'il doit loger 400 prisonniers à la fois dans une prison dont la capacité est d'environ 300 détenus. Il en loge 227 dans des cellules simples, 24 dans 8 cellules à trois occupants, 9 dans les cachots, 140 à 150 dans les 7 quartiers ouverts. Il aménagea aussi deux réduits sous les combles en quartiers confortables pour les vieillards et les invalides.

La surpopulation se fait surtout sentir à partir de 1905. Pour la première fois depuis 28 ans, le nombre des incarcérations à la prison de Montreal approche 4000 (3972). Le 17 avril, le gouverneur dénombrait 400 détenus, soit 150 de plus qu'à la même date l'année précédente. M. Vallée écrivit au shérif Thibaudeau pour lui faire part de ses appréhensions et lui demander de transférer sans délai 100 prisonniers à Québec ${ }^{\prime 2}$ On effectua 13 transferts entre les années 1906 et 1912. En 1911, le nombre total des incarcérations dépassa 6000 alors que l'année suivante la cour envoya 6099 personnes purger une sentence d'emprisonnement à la prison du district de Montréal. Heureusement, en novembre 1912, la nouvelle prison de Bordeaux pouvait recevoir ses 100 premiers détenus.

En plus du problème de la surpopulation, on devait faire face à la prison du Pied-du-courant à des conditions de détention moins qu'admissibles. Ainsi par exemple, l'abbé J.B. Langlois, aumônier de la prison de Montréal, écrivait en 1867, dans une lettre à son supérieur :

D'abord, il n'y a pas de ventilation et puis la petite demi-lumière de la croisée est habituellement fermée et néanmoins déjà de par sa nature insuffisante à fournir un air sain à l'appartement. L'air y est si corrompu que c'est à s'y sentir

11. Premier rapport annuel du Bureau des inspecteurs des prisons, asiles. etc., pour les annees 1867-1868 (1868), in: Documents de la session (1869) vol. 2. doc. 23.

12. Lettre de M. C.A. Vallée à J.R. Thibaudeau, shérif, en date du 17 avril 1905, in : correspondance Vallée. 
le coeur suffoqué quand le matin... on pénètre dans les cachots...

Les prisonniers (des deux sexes) ne sont pas décemment habillés... À leur entrée en prison, on les dépouillait de leurs habits pour ne les faire couvrir que de misérables haillons très insuffisants à les couvrir. Grand nombre d'entre eux étaient même obligés à garder le lit par un geste de pudeur. De plus, les changes sont si rares qu'il y a habituellement de la vermine; le prêtre en exerçant son ministère l'a plus d'une fois senti...

Les femmes toujours en grand nombre, ne peuvent aller au grand air. Il n'y a pas de cour pour elles et, y en eut-il qu'elles seraient privées d'y aller parce que par la disposition des lieux, elles auraient l'occasion de voir les prisonniers, d'être vues par eux et de se parler... ${ }^{13}$

La situation ne s'était évidemment pas améliorée au début du $20^{\circ}$ siècle. Ainsi, dans une lettre au shérif de Montréal, le gouverneur Vallée écrit en 1910 :

Je ne saurais, me semble-t-il avoir un meilleur à propos de faire valoir encore une fois auprès de vous, M. le shérif, combien ça nous devient chaque jour plus difficile de maintenir efficacement la discipline chez nos 400 ou 450 détenus. L'encombrement d'une part, et de l'autre l'impossibilité matérielle de répartir d'abord, puis d'isoler les prisonniers par catégories homogènes se traduisent par un pêle-mêle des plus pernicieux...

La situation qui nous est faite n'est-elle pas plus rassurante au point de vue de l'hygiène qu'à celui de la discipline et de la morale. Surtout en hiver, nos locaux surpeuplés deviennent de véritables foyers morbifiques ${ }^{14}$

Au début du $20^{\circ}$ siècle, le surpeuplement et les conditions de détention ne sont plus les seules à justifier le besoin d'un nouvel établissement. En effet, la prison commence à se détériorer à un rythme accéléré. En février 1902, au cours d'une inspection, $M$. Vallée s'aperçut que tous les encadrements des portes des cellules du quartier numéro 3 (aile nord-est) étaient pourris jusqu'au cour. Poussant plus loin son investigation, il constata que les murs des quartiers 3, 6, 9, et 10 faiblissaient ${ }^{15}$. Le département des Travaux

13. B. Langlois, un aperçu de la prison de Montréal (1867), document manuscrit, archives de la chancellerie, archidiocèse de Montréal.

14. Lettre de C.A. Vallée au shérif Lemieux en date du 22 octobre 1910, in : correspondance du shérif.

15. Lettre de Thibaudeau à A. Brassard, assistant-architecte du gouvernement en date du 18 décembre 1905. 
publics lui envoya M. Brassard, assistant-architecte pour leur compte, pour procéder à l'examen des lieux.

M. Brassard découvrit que non seulement les encadrements des portes n'offraient plus de sécurité, que les fondations s'affaiblissaient mais également que les planchers des cellules du passage devaient être refaits dans le quartier numéro 3.

Pour leur part, les inspecteurs des prisons A.L. de Martigny et J.J.E. Woods constatent en 1904 :

Malheureusement, il semble que plus on répare cette masure, plus elle a besoin d'être réparé...

Le seul remède à apporter à ce déplorable état de choses serait la construction d'une aile supplémentaire à l'édifice...

Les parquets, les escaliers, les boiseries, les charpentes et les armatures en fer tiennent à peine... même si l'intérieur en était démoli et entièrement reconstruit, la prison ne vaudrait pas grand chose en raison de la disposition des annexes qui rend la surveillance des détenus excessivement difficile...

\section{LES ÉTAPES DE LA CONSTRUCTION DE LA PRISON DE BORDEAUX}

La situation déplorable de la prison du Pied-du-courant amena le gouvernement à envisager la construction d'une nouvelle prison dès 1890. Cette année-là, Honoré Mercier, soucieux de mettre un terme à cette situation de la prison de Montréal, suggère à l'Assemblée législative de voter un crédit de 300000 dollars affectables à la construction d'un nouvel édifice ${ }^{16}$.

Puis, en 1891, la dernière année de son mandat, le gouvernement acquit deux terres cultivables de la paroisse du Saut-aux-Récollets. La première achetée pour la somme de 18000 dollars à un dénommé James Inglis Roy, a 3 arpents de front sur 30 de profondeur. Elle est bornée par la rivière des Prairies. La seconde, de même longueur, mais de largeur moindre ( 2 arpents) propriété de M. Harry Mc Laren, ne coûta que 8500 dollars. C'est sur ce site que sera construite la prison de Bordeaux.

Mais en décembre de la même année, l'honorable Mercier perdit le pouvoir, les projets de construction de Bordeaux furent

16. Discours de l'honorable M. Mercier, premier ministre de la province, prononcé à l'assemblée législative de Québec le 21 février 1890 en réponse à la critique faite par M. Desjardins, député de Monmorency à l'exposé budgetaire (1890 p. $40-41$ ). 
mis en veilleuse. Ce n'est qu'au retour des libéraux, en 1905, que le projet de construction d'une nouvelle prison sera repris.

Cette année-là, le gouverneur Vallée demanda un congé de trois mois au shérif Thibaudeau pour aller faire un séjour en Europe. Il disait vouloir profiter de son séjour «pour faire des études spéciales sur les différents régimes pénitentiaires ${ }^{17}$ ". Le gouverneur Vallée fut absent du 9 septembre au 7 décembre $1905^{18}$.

Les inspecteurs de prison, le shérif et le gouverneur de la prison de Montréal exercent de constantes pressions sur le gouvernement de la province pour qu'ils pourvoient au remplacement de la maison de détention. En 1906, les journaux firent échos à leurs protestations ${ }^{19}$.

De fait, le 25 juin 1906, MM. J.O. Marchand et R.A. Brassard offrirent leurs services pour la préparation des plans et devis. Le 21 août, le gouvernement leur confia la direction des travaux.

... L'honorable ministre des Travaux publics et du travail m'a donné instruction de vous dire la charge de préparer les plans et devis (spécification) d'une nouvelle prison commune pour le district de Montréal... ainsi que la surveillance des travaux se rapportant à la construction de cet édifice, la vérification des comptes relatifs à cette entreprise, etc., et cela aux conditions suivantes :

1. pour la préparation des plans et devis... il vous sera accordé une commission de $4 \%$ sur le coût de l'entreprise, si celle-ci doit être une somme moindre de 500000 dollars, et $3 \frac{1}{2} \%$ sur tout excédent de cette somme de 500000 dollars.

2. sur les pourcentages ou honoraires qui précèdent, il vous sera payé un acompte de $1 \frac{1}{2} \%$ pour les prix des plans d'ensemble, lors de leur livraison...

3. la balance de la commission sera payable au fur et à mesure que les travaux avanceront...

4. vous devez fournir au département des Travaux publics et du travail les plans et devis...

5. toutes les dépenses à encourir pour frais de voyage ou autres... seront à votre charge... ${ }^{20}$

17. Lettre de C.A. Vallée au shérif Thibaudeau en date du 29 août 1905.

18. Lettre du shérif Thibaudeau à $C$. Lanctot, assistant-procureur général en date du 6 décembre 1905.

19. The necessity for a new jail, in : Daily witness, Montreal Saturday 5 mai 1906 , vol. XLVIl, no 106.

20. Lettre de E. Gagnon, secrétaire du département des Travaux Publics aux architectes Marchand et Brassard, datée de Québec le 21 août 1906, in : Document de la session (1911), vol. 44, no 4, doc. 67. 
M. Weir, le nouveau ministre des Travaux Publics, demanda à l'assemblée le 8 mars 1907 d'adopter une loi permettant l'édification d'un nouveau bâtiment. Le 14 mars, on adopta «la loi concernant la construction d'une nouvelle prison dans le district de Montréal ${ }^{21}$.

Aux termes de cette loi, la prison de Montréal devait être érigée sur la propriété du gouvernement achetée en 1891 aux Saut-aux-Récollets. Le coût de cet établissement pénal ne devait pas excéder 550000 dollars (Art. 3)

Le 16 septembre, M. Jean-Baptiste Pauzé, constructeur se présente devant le notaire Arthur Écrément pour signer le premier contrat de construction. L'entrepreneur s'engage à débuter les travaux le ou avant le $1^{\text {er }}$ octobre et à les poursuivre sans interruption. Il devait terminer le soubassement de toutes les ailes et la superstructure des ailes A.E. et F. au plus tard le $1^{\text {er }}$ mai 1910.

\section{M. J.A. VALLÉE}

M. J.A. Vallée, l'un des principaux artisans de la construction de la prison de Montréal mérite d'être présenté.

Charles A. Vallée fut gouverneur de la prison du Pied-du-courant du 18 mai 1891 jusqu'à la fermeture en 1912. Il fut aussi durant les premières années, gouverneur de la prison de Bordeaux. Ancien zouave pontifical - il avait fait la campagne de Rome en 1870 - il était très au fait des questions pénales, et avait des idées qui correspondaient, comme nous le verrons, à celles de son époque.

Lorsqu'en 1906, le procureur général lui demandant s'il croyait que les prisonniers devraient dans la nouvelle prison être traités en commun... ou d'après le système adopté par plusieurs pays d'Europe, notamment la France et la Belgique, M. Vallée répondit que la prison commune doit servir à la fois de maison d'arrêt pour les prévenus, de maison de justice pour les inculpés, de maison de correction pour les condamnés aux travaux forcés et de prison pour les autres détenus et aliénés. Dans ces circonstances. il ne pouvait opter pour l'isolement cellulaire tel que pratiqué en Belgique, bien qu'il en soit un partisan très zélé.

Il n'y a pas à en douter, le système cellulaire est bien le remede le plus efficace au débordement de la corruption

21. Statut du Québec, Edouard VII, chap. 36. 
qu'engendre la promiscuité dans nos prisons, lui seul peut commencer l'ouvre de la moralisation mais à une condition expresse : c'est qu'il n'y ait pas de chômage.

Le principal motif qui le poussait malgré tout à favoriser la prison cellulaire mixte - c'est-à-dire un régime où les prisonniers travaillent en commun le jour et sont isolés la nuit - était la crainte de voir les prisonniers manquer de travail.

Dans notre pays où les unions ouvrières règnent en souveraines; où de toutes parts on s'oppose avec tant d'acharnement au travail, même pénal, fait dans les prisons, est-il possible d'établir le système cellulaire pour tous les détenus indifféremment? Je réponds catégoriquement non, car le système pêcherait par la base même : le manque de travail.

Je serais pourtant favorable à un quartier cellulaire pour les inculpés, prévenus et accusés, car ils ont des droits incontestables en vertu de leur innocence présumée ou possible...

M. Vallée avait aussi une conception particulière mais très comtemporaine des délinquants, comme le démontre son insistance à avoir une ventilation adéquate dans la nouvelle prison.

... S'il y a une place où l'aération doit être parfaite c'est bien dans une prison car, chose singulière, le criminel comme l'aliéné semblent exhaler de leurs corps une odeur «sui generisn. Ces émanations se répandent et s'imprègnent partout, à moins que l'air ne soit continuellement renouvelé...

Le gouverneur de la prison du Pied-du-courant a aussi, comme nous l'avons vu, une option peu favorable des unions ouvrières ainsi que des étrangers.

... Et cet état de choses, je dois l'ajouter, me semble compliquer d'autant plus la situation que la mentalité de nos prisonniers est elle-même plus différente aujourd'hui, de ce qu'elle était autrefois. Il y pointe maintenant un esprit de revolte pour ainsi dire chronique contre l'autorité sous toutes ses formes... Je n'ai sans doute pas à déterminer ici jusqu'où cette agressivité particulière et généralement imputable aux doctrines subversives que des fauteurs de grèves ouvrières d'unionismes internationalistes (sic) ou de communisme obligatoire (sic) ont importé chez nous de la vieille Europe; mais, personnellement, je suis bien convaincu que, entre cette mentalité nouvelle que je signale chez nos détenus et la vulgarité des doctrines précitées, il doit exister autre chose de plus substantielle que de simples rapports de coïncidence éventuelle... ${ }^{22}$

22. Lettre C.A. Vallée au shérif Lemieux en date du 22 octobre 1910. in: correspondance du shérif. 


\section{E. LES ARCHITECTES}

En août 1906, le gouvernement confia la charge de préparer les plans et devis aux architectes Marchand et Brassard. M. Joseph Omer Marchand appartenait au bureau Marchand et Haskell de la rue St-Jacques à Montréal. Né à Montréal le 28 décembre 1873, il commença ses études avec les architectes Perrault et Mesnard. À 18 ans, il s'embarqua pour l'Europe et s'inscrivit à l'École des Beaux-Arts de Paris où il étudia pendant 10 ans. Il revint avec un diplôme du gouvernement français.

En 1906, alors qu'il était âgé à peine d'une trentaine d'années, il avait déjà construit l'hôpital Notre-Dame, celui des contagieux, la bibliothèque et la chapelle du grand séminaire, l'église de Sainte-Cunégonde, la maison-mère et le noviciat des religieuses de la congrégation de Notre-Dame. Par la suite, il dressa les plans d'une trentaine d'édifices, dont la Cour juvénile et la prison des femmes du Québec. Membre de l'association des architectes depuis 1902, il en devint président en 1927. Il mourut le 12 juin 1936 à l'âge de 63 ans.

M. Raoul Adolphe Brassard (18 -1927) n'appartint jamais, semble-t-il, à l'association des architectes. Tout nous laisse croire qu'il participa à l'élaboration des plans de Bordeaux en tant qu'assistant-architecte. Ce titre lui est d'ailleurs conféré dans un document en date du 18 décembre $1905^{23}$. Il participa pour le compte du ministère des Travaux publics aux travaux de construction des prisons de Valleyfield et de Bordeaux, aux réparations du Palais de Justice et du Parlement d'Ottawa ${ }^{24}$. Il mourut le 15 septembre 1927 à la suite d'un accident. À cette époque, il était secrétaire de la Commission des tramways de Montréal.

\section{F. BORDEAUX 1913}

La prison de Bordeaux a fait couler beaucoup d'encre et mérita de sévères critiques au gouvernement de l'époque. Les coûts et la durée de la construction créerent presque un scandale politique. Ainsi d'une part, la loi concernant la construction d'une nouvelle prison dans le district de Montréal de 1907 prévoyait un coût maximal de 550000 dollars alors qu'en 1912, le trésor public avait

23. Lettre de Thibaudeau à A. Brassard, assistant-architecte de l'époque du gouvernement du 18 décembre 1905 .

24. Etat des comptes publics de la province de Québec (1903 p. 155). 
déjà dépensé plus de deux millions et demi pour la construction ${ }^{25}$. D'autre part, la même loi prévoyait que le gros de la construction devrait être terminé le $1^{\text {er }}$ mai 1910 mais en 1911 , le journal le Devoir, pour ne citer que lui, taxa de "bonne blague" l'affirmation de M. Taschereau, ministre des Travaux publics, selon laquelle la fin des travaux se situerait en février 1912.

... Le mur d'enceinte ne sort pas encore de terre. Et l'on ne bâtit pas un mur pareil, qui doit entourer un mur si vaste, dans le temps de le dire. Quant à changer les prisonniers de logis sans prendre les précautions nécessaires, le ministre n'oserait pas, au moins avant les élections et $M$. le gouverneur Vallée lui-même, tout pressé qu'il soit de villégiaturer, tout désireux qu'il puisse être de doter le district de Montréal d'un château digne de la renommée et du mérite de ses hôtes, ne voudrait probablement pas risquer l'aventure. L'air est la suprême préoccupation des grands hygiénistes; et l'on ne songerait sans doute pas à priver MM. les voleurs ou assassins de cet élément essentiel à leurs poumons. Or, qui sait sí ces Messieurs que l'on va transformer en châtelains ne se croiraient pas tout permis, une fois rendus à Bordeaux. Le luxe est une habitude qui développe le goût des voyages...

Alors, à l'œuvre sur la prison, dans la prison, autour de la prison! Aujourd'hui 100 hommes, la semaine prochaine 800 hommes. À l'œuvre! À l'œuvre! Il faut finir la prison de Bordeaux. C'est $\mathbf{M}$. le gouverneur qui le demande, c'est $\mathbf{M}$. Alexandre Taschereau qui le veut... (29 mai).

Ce n'est que le 18 novembre 1912 que Bordeaux reçut ses 100 premiers détenus.

En 1913, la prison de Bordeaux comprend 558 cellules. Elle est entourée de deux murs de $25 \times 18$ pieds de hauteur entre lesquels se trouve le chemin de ronde où quatre sentinelles montent la garde nuit et jour. Le chemin dit de "service» court le long du petit mur à l'intérieur. Construite selon le style «Pennsylvanien» propre aux pénitenciers où l'on pratiquait l'isolement cellulaire, elle affecte la forme d'un astérisque. On y accède uniquement en traversant le corps de garde. Celui-ci avec ses meurtrières, ses crénaux et ses massives portes de fer rappelle les forteresses d'antan et donne à l'ensemble une allure très imposante. La tour centrale de la prison vers laquelle convergent les 6 ailes de la prison est surmontée d'un dôme dont la coupole est située à 150 pieds au-dessus du sol.

25. Journaux de l'Assemblée législative (1912) vol. 46 p. 125-126. 
La chapelle catholique occupe le sommet de la tour ( $3^{e}$ étage). Le prêtre officie au centre de la pièce et les détenus assistent à l'office divin dans des stalles aménagées dans les encoignures des ailes, entre de grandes baies bardées qui donnent directement sur ces ailes. La rotonde, le cœur de l'établissement, est sise au rez-de-chaussée au milieu de la tour. Chaque aile est désignée par une lettre : A, B, C, D, E, F. La première réservée à la prévention contient 162 cellules, les quatre suivantes 198. La sixième n'en a pas. On y trouve les parloirs, les infirmeries, les appartements de l'aumônier catholique, etc.

Il existe trois types de cellules : celles des condamnés à mort, des prévenus et des détenus. Les premières au nombre de quatre sont contiguës par paire et sises à l'avant de la prison de chaque côté de l'infirmerie. Les cellules des prévenus de l'aile A mesurent $8 \times 11 \times 9$ pieds. Ceux-ci restant enfermés toute la journée sauf au moment de la marche quotidienne, les architectes ont voulu leur offrir un cubage d'air suffisant et un confort supérieur à cause de la présomption d'innocence qui pèse sur eux. Les cellules ordinaires ont $6 \frac{1}{2} \times 11 \times 9$ pieds de hauteur. Une lourde porte métallique en obstrue l'entrée. Un judas permet aux gardiens d'observer les détenus qui s'y trouvent. Elles sont actionnées automatiquement au moyen de leviers.

Bien que dessinée selon le style «Pennsylvanien», c'est-à-dire étoilée avec des cellules disposées le long du mur, la prison de Bordeaux n'est pas destinée à l'emprisonnement cellulaire. Désireux de mettre fin à la promiscuité, source de corruption dans les prisons communes, M. Vallée opta pour l'emprisonnement individuel. Il ne put cependant instaurer un régime cellulaire par peur de manque de travail. Il se résigna donc à adopter un système Auburnien mais exigea une prison construite dans le plus pur style Pennsylvanien. Sur un croquis d'époque, on retrouve même des préaux individuels disposés en cercle comme on en retrouve dans les prisons Pennsylvaniennes construites en Europe ${ }^{2 i}$.

L'admiration que portait $M$. Vallée pour le système belge nous porte à croire que la prison de Bordeaux est d'inspiration belge. On a aussi souligné à l'époque, la ressemblance entre la nouvelle prison de Bordeaux et celle de Saint-Gilles de Bruxelles et de Verviers. 
La prison bastille de Saint-Gilles a été construite entre les années 1876 et 1883 par le célèbre architecte Derre, qui écrivit sur l'art d'ériger des prisons, - pour desservir une population de 600 détenus. La prison de Saint-Gilles ressemble beaucoup à la prison de Bordeaux mais tout pénitencier de type Pennsylvanien fait songer à un autre pénitencier du même style. Des études plus approfondies seraient utiles pour voir la similarité entre les deux édifices. Par contre, il semble que l'on a retenu de Verviers la disposition de la chapelle au sommet de la tour centrale.

Soulignons enfin que nous n'avons jamais retrouvé, lors de nos recherches, quelque indication que ce soit ou quelque référence que ce soit à la prison de Fresne en France, construite en 1898. L'architecture de cette prison, d'une conception toute nouvelle, sous forme de "téléphonepole» a été construite durant le séjour de $\mathbf{M}$. Marchand en France et aurait pu être au centre des discussions lorsqu'on a construit Bordeaux. De fait, ce type d'architecture a influencé grandement celui de plusieurs prisons américaines du début du $20^{\mathrm{e}}$ siècle.

Ces quelques notes de recherche ne sont qu'une première étape dans la cueillette des données sur l'histoire de la réaction pénale et sociale au Québec. Elles contribuent, espérons-nous, à accumuler les informations qui nous permettront de mieux comprendre et interpréter quelles conceptions on s'est faites chez nous du délinquant, de la criminalité et de la réaction pénale.

\section{BIBLIOGRAPHIE}

BOYER, R. (1966) : les Crimes et les châtiments au Canada français, Montréal, Le Cercle du livre de France.

CRAWFORD. W., (1835) : (réédition 1969) Report of the Penitentiaries of the United States, Montclair, New Jersey, Patterson Smith.

DE BEAUMONT, G. et A. DE TOCQUEVILLE (1833) : Du système pénitentiaire aux Etats-Unis et son application en France, Paris, Librairie H. Fournier Jeune.

DE MARTIGNY, A.L. et J.J.E. WOODS (1904) : Trente-quatrième rapport des inspecteurs de prisons et d'asiles de la province de Québec pour l'année 1903, in : Documents de la Session (1905), vol. XXXV1il, no 2, doc. 4.

MONDELET, D. et J. NEILSON (1835) Rapport des commissaires chargés de visiter les pénitenciers d'Amérique, in : $X I V$ volume des journaux de la Chambre d'assemblée de la province du Bas-Canada, Appendice $C$.

NELSON, W.D., J.C. TACHE, D.A.E. MACDONALD, J. LANGTON et E.A. MEDERITH (1859) : Rapport préliminaire du bureau des inspecteurs d'asiles, prisons, etc. 1859, in : Documents parlementaires (1860), vol. XVIII, no 4, doc. 32 . 
NORMANDEAU, A. (1970) Politique et réforme pénitentiaire : le cas de la France 1789-1875), Revue de science criminelle et de droit pénal comparé, no 3,605-621.

OUIMET, R. (Prés.) (1969). Rapport du Comité canadien de la réforme pénale et correctionnelle, Ottawa, Information Canada.

RODIER, E. (12-3-1836), Rapport du Comité spécial chargé de prendre en considération le rapport des commissaires nommés pour visiter les pénitenciers Américains, in : Continuation de l'appendice au XIV volume des journaux de la Chambre d'assemblée du Bas-Canada, (1835-36), vol. 111, appendice F.F.F. section C. 